\title{
Near-field radio emission induced by extensive air showers
}

\author{
Daniel García-Fernández ${ }^{* a}$, Didier Charrier ${ }^{a}$, Richard Dallier ${ }^{a, c}$, Antony Escudie $^{a}$, \\ Alain Lecacheux ${ }^{b}$, Lilian Martin ${ }^{a, c}$, Benoît Revenu ${ }^{a, c}$, Matias Tueros ${ }^{d}$ \\ ${ }^{a}$ Subatech, Institut Mines-Télécom Atlantique, CNRS, Université de Nantes, Nantes, France \\ ${ }^{b}$ CNRS-Observatoire de Paris, Meudon, France \\ ${ }^{c}$ Unité Scientifique de Nançay, Observatoire de Paris, CNRS, PSL, UO/OSUC, Nancçay, France \\ ${ }^{d}$ Instituto de Física La Plata, CONICET, CCT-La Plata, La Plata, Argentina \\ E-mail: daniel.garcia-fernandez@subatech.in2p3.fr
}

\begin{abstract}
The measurement of the electric field created by cosmic-ray induced air showers is nowadays a well-established technique. Due to technical limitations, the low-end part of the frequency spectrum of the field has not been thoroughly exploited or understood, even though some experiments have indicated a large electric field at low frequencies. In this work, we present a new equation for the electric field of a particle track in time-domain valid for all frequencies, and therefore suited for the treatment of the near-field regime. After that, we calculate the low frequency radio signal of extensive air showers in the far-field and near-field regimes using a new version of the SELFAS Monte Carlo code that includes our equation.
\end{abstract}

35th International Cosmic Ray Conference - ICRC2017

10-20 July, 2017

Bexco, Busan, Korea

\footnotetext{
${ }^{*}$ Speaker.
} 


\section{Introduction}

The low flux of cosmic rays with energies above $\sim 10^{15} \mathrm{eV}$ impedes their direct measurement, so we are forced to detect them indirectly via the extensive air showers (EAS) created upon the interaction of a primary cosmic ray with the atmosphere. The particles composing the EAS can give us some information on the properties of the primary cosmic ray. There are three main detection techniques - the detection of the particles arriving at ground level with Cherenkov detectors or scintillators, the detection of the fluorescence light emitted by the atmosphere after the passage of the charged particles of the EAS, and the detection using radio antennas.

Radio detection is nowadays a well-established technique [1] that allows us to infer the properties of the primary cosmic ray by measuring the electric field created by the charged particles in the EAS. The electric field is created, at first order, by the current induced by the deflection of the secondary particles of the EAS in the geomagnetic field, a mechanism known as the geomagnetic effect [2]. A subdominant mechanism, called the Askaryan effect, is also present, and it consists in the creation of a radiation electric field by the excess of negative particles in the shower [3,4].

While every currently operating experiment (for instance, CODALEMA [6], AERA [7] or Tunka-Rex [8]) measures the electric field above $20 \mathrm{MHz}$, several experiments have detected an inequivocal low-frequency electric signal (below $20 \mathrm{MHz}$ ) coming from EAS, for instance [9, 10], although their properties have not been properly explained with a known mechanism. A review of many low-frequency experiments can be found in [5], and they all agree in the existence of a lowfrequency counterpart with an amplitude higher than the usual emission above $20 \mathrm{MHz}$. Moreover, the coherent deceleration of the shower front, that is, the sudden stop of the shower particles when they arrive to the ground, has been proposed in [5], but also in [9], as the possible source of the low-frequency signal. We will call this the sudden death mechanism.

In order to measure the electric field of an EAS at low frequencies, the EXTASIS experiment has been set up at the Nançay radio observatory [11], with an effective frequency band spanning from 1.7 to $3.7 \mathrm{MHz}$. One of the main theoretical problems that arises is the accurate calculation of the electric field. Standard codes for the calculation of the electric field, such as SELFAS, ZHAireS or CoREAS, calculate the electric field of the shower in far-field, that is, when the wavenumber of the emission $k$ and the distance from emitter to observer $R$ verify $k R=2 \pi R / \lambda \gg 1$. If we expect an important emission from the lower part of the shower (as in the sudden death mechanism), and if the antennas are situated at a distance smaller than $100 \mathrm{~m}$ from the shower core, we have that at 1 MHz: $k R=2 \pi 100 / 300 \sim 2$, indicating that near-field effects could be important for antennas near the shower core.

In this work, we will present a formula for the field of a particle track that does not require the far-field approximation, compare it with a previous well-known formula, and then introduce it in the SELFAS Monte Carlo code to apply it to calculate the electric field of an EAS at low frequencies.

\section{Electric field for a particle track}

We define a particle track as the trajectory of a particle that begins at rest, gets suddenly accelerated up to a velocity $\mathbf{v}$ at $t=t_{1}$, travels in a straight line at constant speed and then is 
suddenly stopped at $t=t_{2}$. The track is the foundation of practically all the Monte Carlo codes that calculate the electric field of EAS in a microscopic way by discretising a particle trajectory in straight segments, an then calculate the total field as the superposition of the fields created by the tracks. Assuming an homogeneous, non-magnetic medium, we can write the electric field as [12]

$$
\mathbf{E}(\mathbf{x}, t)=\frac{1}{4 \pi \varepsilon} \int \mathrm{d}^{3} x^{\prime}\left\{\left[\frac{\rho\left(\mathbf{x}^{\prime}, t_{\mathrm{ret}}\right) \mathbf{r}}{R^{2}(1-n \beta \cdot \mathbf{r})}\right]_{\mathrm{ret}}+\frac{n}{c} \frac{\partial}{\partial t}\left[\frac{\rho\left(\mathbf{x}^{\prime}, t_{\mathrm{ret}}\right) \mathbf{r}}{R(1-n \beta \cdot \mathbf{r})}\right]_{\mathrm{ret}}-\frac{n^{2}}{c^{2}} \frac{\partial}{\partial t}\left[\frac{\mathbf{J}\left(\mathbf{x}^{\prime}, t_{\mathrm{ret}}\right)}{R(1-n \beta \cdot \mathbf{r})}\right]_{\mathrm{ret}}\right\},
$$

where $n=\sqrt{\varepsilon}$ is the refractive index of the medium, $\mathbf{r}$ is the normalised vector of the line of sight, $R$ the distance between observer and emitter, $c$ is the speed of light in the vacuum, $\mathbf{v}$ is the velocity and $\beta=\mathbf{v} / c$ and the calculation is performed in retarded (or emission) time. No far-field frequency approximation has been assumed whatsoever, which means that Eq. (2.1) is suitable for obtaining the electric field at low frequencies and short distances as well, as long as the trajectory is accurately represented. The only caveat is that we must choose a charge density that verifies charge conservation, that is, the total charge in space must be conserved, otherwise the field will not be a solution of Maxwell's equations and the field will not be correct, in general. We can write such a charge density for a single track as

$\rho(\mathbf{x}, t)=q \delta^{(3)}\left(\mathbf{x}-\mathbf{x}_{1}\right) \Theta\left(t_{1}-t\right)+q \boldsymbol{\delta}^{(3)}\left(\mathbf{x}-\mathbf{x}_{1}-\mathbf{v}\left(t-t_{1}\right)\right) \Theta\left(t-t_{1}\right) \Theta\left(t_{2}-t_{1}\right)+q \boldsymbol{\delta}^{(3)}\left(\mathbf{x}-\mathbf{x}_{2}\right) \Theta\left(t-t_{2}\right)$,

with $q$ the charge of the particle. The current is readily written as

$$
\mathbf{J}(\mathbf{x}, t)=q \boldsymbol{\delta}^{(3)} \mathbf{v} \Theta\left(t-t_{1}\right) \Theta\left(t_{2}-t_{1}\right) .
$$

Contrary to what one could expect, the addition of the first and third terms in Eq. (2.2) does create a radiation electric field. Physically, this is due to the fact that an acceleration field is not the same as a charge creation field, the latter being physically impossible without an opposite charge being created at the same time. Mathematically, we have a field of the form

$$
\mathbf{E}_{\text {cons }, 1}(\mathbf{x}, t)=\frac{q}{4 \pi \varepsilon} \frac{\mathbf{r}_{1}}{R_{1}} \frac{\partial}{\partial t} \Theta\left(t_{\text {ret }}-t_{1}\right)
$$

and an analogous term for the deceleration. The field in Eq. (2.4) represents an impulse field. We will give more details of this calculation in an upcoming paper.

\subsection{Calculation of field and comparison with far-field (ZHS) formula in time domain}

We will now consider an electron track and calculate numerically the electric field with the help of Eq. (2.1) using the densities in Eqs. (2.2) and (2.3), but adding a positive static charge at position $\mathbf{x}_{1}$ so that the total charge in space is zero, and the static electric field at large distances is zero (this does not affect the generality of our result). The numerical step in time is taken to be $\Delta t=0.1 \mathrm{~ns}$ and the numerical derivative is a two-point derivative using the values of the two adjacent points and calculating their difference.

Although our main interest is to calculate the field from EAS, formulas involving refractive indexes are better studied within dense media. Because of this, let us take a $1.2 \mathrm{~m}$ long electron track along the $z$ axis at $v \sim c$ in an homogeneous, non-magnetic medium with refractive index $n=1.78$ (akin to that of deep Antarctic ice), and let us place an observer at a distance of $10 \mathrm{~m}$ 
from the centre of the track and at an angle of $\theta_{C}+10^{\circ}$, with $\theta_{C}=\operatorname{acos} \frac{1}{n \beta}$ the Cherenkov angle. We show in Fig. 1 (left) that the pulsed fields for the acceleration and deceleration of the track in this configuration using Eq. (2.1) are the same as those obtained with the well-known ZHS time formula [13] (radiation field calculated in the Coulomb gauge). Not taking into account the charge conservation results in an unphysical field (green line) that differs from the Coulomb gauge calculation. Placing an observer at $100 \mathrm{~m}$ yields a similar result (see Fig. 1, right), this time within the far-field zone, where no field is seen between the two acceleration and deceleration pulses. Again, not taking into account the charge conservation results in an incorrect radiation field.
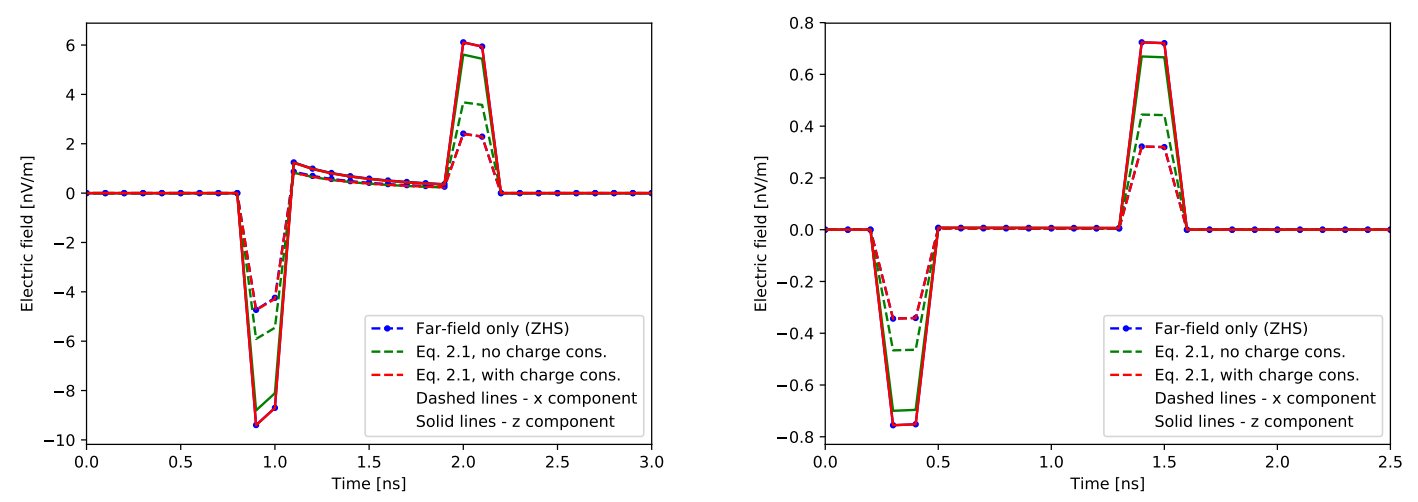

Figure 1: Electric field (in different approaches) created by a $1.2 \mathrm{~m}$ long electron track travelling along the $z$ axis, as a function of time. Observer is placed at $\theta_{C}+10^{\circ}$, in a medium with $n=1.78$. Left: $R=10 \mathrm{~m}$. Right: $R=100 \mathrm{~m}$. Times have been arbitrarily offset. See text for details.

\subsection{Calculation of field and comparison with an exact formula in frequency domain}

Eq. (2.1) can be transformed to frequency domain. This transformation, besides allowing us to study our instrumental frequency range, it helps us to overcome the numerical divergences at the Cherenkov angle, since in frequency domain we can use the retarded time alone and so the divergent denominators in Eq. (2.1) are not present. The field for a track in the frequency domain can be written as

$$
\begin{aligned}
\mathbf{E}(\mathbf{x}, \omega) & =\frac{q}{4 \pi \varepsilon}\left\{\int_{t_{1}}^{t_{2}} \mathrm{~d} t e^{i \omega t} \frac{e^{i k R}}{R} \mathbf{r}\left[\frac{1}{R}-\frac{i \omega n}{c}\right]+\sum_{j=1,2}\left[(-1)^{j} e^{i \omega t_{j}} e^{i k R_{j}} \mathbf{r}_{j}\left(\frac{n}{c R_{j}}+\frac{i}{\omega R_{j}^{2}}\right)\right]\right\} \\
& +\frac{i \omega \mu_{0} q}{4 \pi} \int_{t_{1}}^{t_{2}} \mathrm{~d} t e^{i \omega t} \frac{e^{i k R}}{R} \mathbf{v}
\end{aligned}
$$

Eq. (2.5) looks similar to an equation for the exact field of a track presented in [14], which has been proven to be equivalent to the usual radiation field in the far-field regime and to reproduce the Cherenkov radiation for an infinite track. Eq. (2.5) and the equation in [14] can be derived from Maxwell's equations with the same charge and current densities, and are therefore equivalent. We show an example of this equivalence in Fig. 2 (left). Since Eq. (2.5) is equivalent to an exact formula in frequency domain, its time domain counterpart Eq. (2.1) is then a correct implementation of the exact formula of a track in time domain. 

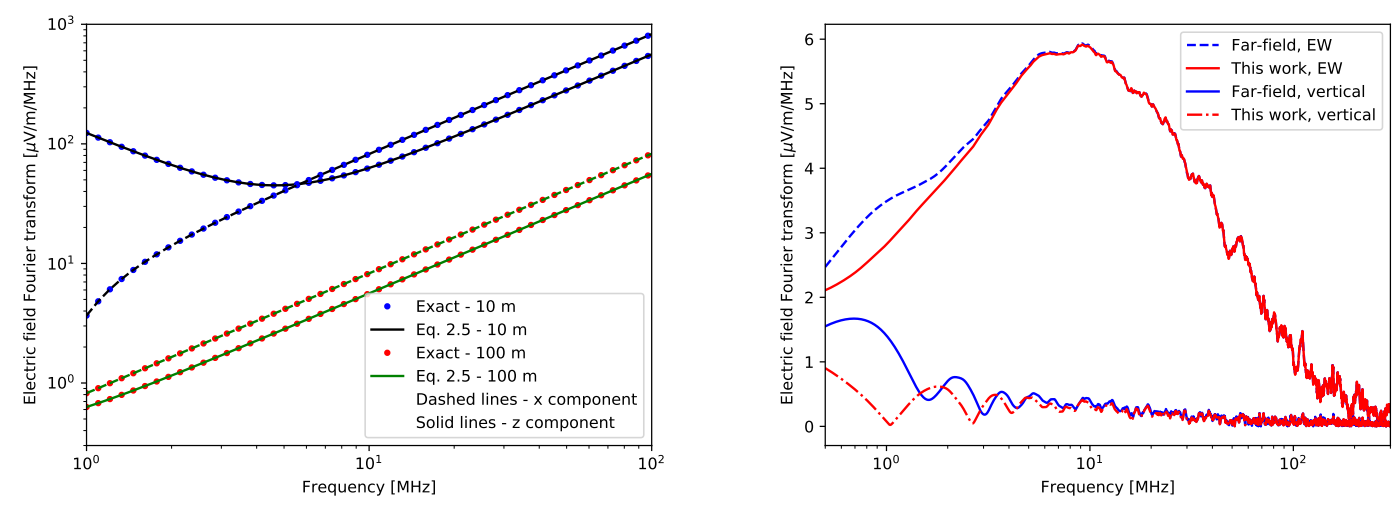

Figure 2: Left: Fourier transform of the electric field as a function of frequency for the same track as in Fig. 1. The observer is placed at the Cherenkov angle $(n=1.78)$. The field has been calculated with Eq. (2.5) and the exact calculation in [14]. Right: Fourier transform amplitude of the electric field created by a $1 \mathrm{EeV}$ proton-induced vertical EAS. The antenna is placed $200 \mathrm{~m}$ East of the shower core. See text for details.

\section{Near-field emission of EAS using the code SELFAS}

\subsection{Implementation in the SELFAS Monte Carlo code}

SELFAS [12] is a code for the calculation of the electric field of an EAS that operates by sampling the particles of the shower, simulating their propagation in the atmosphere for a given depth, and then calculating the field created by the trajectory modelled as a collection of particle tracks. It includes all the state-of-the-art mechanisms for particle propagation and field emission, and it has been recently upgraded with the most realistic available description of the atmosphere using the Global Data Assimilation System (GDAS) [15, 16].

In order to embed Eq. (2.1) into SELFAS, we made the following assumptions:

- The static Coulomb field created after the particles have been stopped is not detectable, since the media (atmosphere and soil) will tend to eliminate all local charge excess and regain electrostatic equilibrium. We will not include static fields, as a consequence.

- When a track reaches ground, we can calculate its field as the sum of the field of one track that stops at the boundary and another one that immediatly accelerates at nearly the same place. This approach can explain transition radiation [17]. In this case there are three contributions to the electric field:

- The direct contribution (as if there was no boundary) that we are able to calculate at all frequencies with Eq. (2.1).

- The reflected (far-field) and surface wave contribution (can be near or far-field) to the electric field, that we will not calculate. The effect of reflection and far-field surface waves in a detector can be taken into account knowing the emission pattern of our antenna with the ground present, by the theorem of electromagnetic reciprocity, without the need of calculating the reflected EAS field at the antenna. 
- The underground transmitted component. Given the large attenuation of radio waves inside soil, we will ignore this contribution.

In other words, we will calculate the direct, near-field regime electric field of an EAS.

\subsection{Results for the simulated showers}

We have simulated a vertical proton-induced $1 \mathrm{EeV}$ shower at the Nançay site (altitude $180 \mathrm{~m}$ ) and placed antennas east of the shower core. We show in Fig. 2 (right), the comparison between the far-field spectrum and the spectrum calculated with Eq. (2.1) (same shower), for the East-West and vertical components of an antenna located $200 \mathrm{~m}$ East of the shower core. It is clearly seen that for the East-West component, both calculations agree for frequencies above $\sim 10 \mathrm{MHz}$, which was expected $(k R \gg 1)$. However, below $10 \mathrm{MHz}$ both approaches differ for both polarisations and we must use Eq. (2.1) if we want to accurately depict the electric field. In this case, the near-field effects tend to decrease the amplitude of the electric field.

We can find in Fig. 3 (left) the filtered ( $<5 \mathrm{MHz}$, sixth order low-pass Butterworth) vertical pulses for two antennas located East of the shower core. We find that the second pulse seen in Fig. 3, related to the sudden death of the shower [5] is always present regardless of whether we take into account or not the near-field effects, albeit the pulse seems to be a bit smaller in the near-field case (5\% in this example). Since it is related to the deceleration of the shower front, the arrival time of this pulse to the detector should be proportional to the distance $d$ between antenna and shower core $(t \sim d / c$, if we take the arrival of the shower at ground level to be $t=0)$, something evidenced in Fig. 3 where the antenna at $500 \mathrm{~m}$ sees this pulse after the antenna at $200 \mathrm{~m}$ (with the correct relative delay of $(500-200) / c=1000 \mathrm{~ns}$. In contrast, what is drastically changed at low frequencies is the vertical component of the principal pulse (geomagnetic and charge excess). Even the filtered EW polarisation exhibits non-negligeable differences, see Fig. 3 (right).
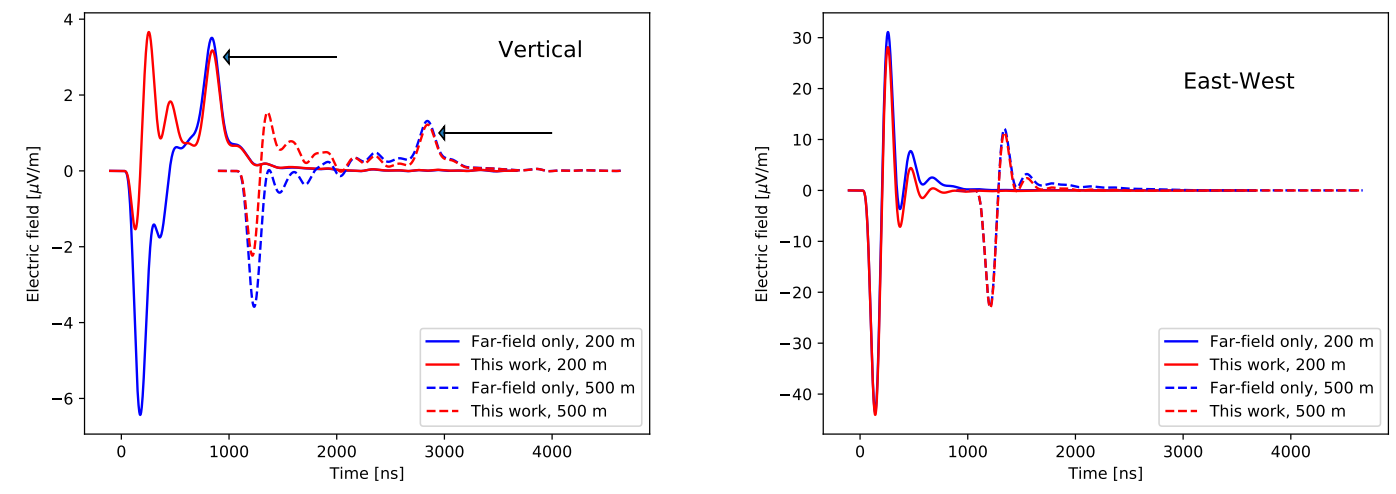

Figure 3: Electric field created by a $1 \mathrm{EeV}$ proton-induced vertical shower for antennas East of the shower core, as a function of time. Times have been arbitrarily offset. The traces have been numerically transformed to frequency domain, filtered using a low-pass sixth order Butterworth filter with a cut frequency of $5 \mathrm{MHz}$ and then transformed back to time domain. The sudden death field (indicated by the arrows) is visible after the principal pulse in each trace. See text for details. Left: Vertical component. Right: East-West component. 
In order to know what order of magnitude should be expected from the so far undetected sudden death pulse (SDP), we calculate its amplitude for a simulated shower. We show in Fig. 4 the filtered amplitudes for proton-induced vertical showers at several distances from the core, as a function of the energy of the primary proton. At $300 \mathrm{~m}$ and $1 \mathrm{EeV}$, the SDP is $\sim 1 \mu \mathrm{V} / \mathrm{m}$. These results are just $\sim 10-20 \%$ lower than those obtained with the standard far-field formula in [5]. The SDP amplitude is proportional to the primary energy.

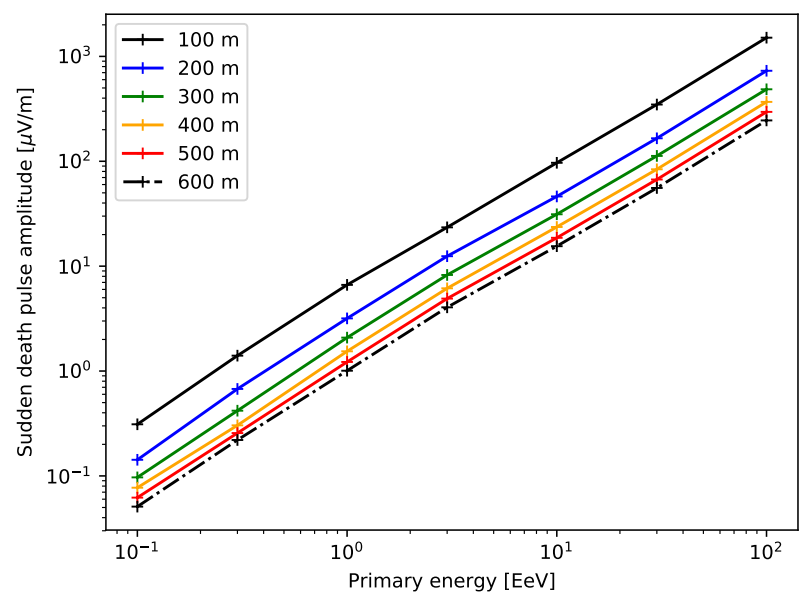

Figure 4: SDP amplitude (filtered as in Fig. 3) for the vertical polarisation, created by vertical proton showers, as a function of the primary energy for different core distances. The antennas are located East of the shower core at the distances specified in the legend.

The sudden death pulse is more prominent at sites with higher ground altitude, where the ground is closer to the shower maximum. This is precisely what we show in Fig. 5 for a $5 \mathrm{EeV}$, $30^{\circ}$ shower at 180 (left) and 2650 (centre) $\mathrm{m}$ of altitude (as in GRAND [18]), where the SDP is larger for the latter case. We also show in Fig. 5 (centre) that, for an antenna at $200 \mathrm{~m}$, the vertical component filtered in the $[5,50] \mathrm{MHz}$ band presents similar results for the far-field approximation and for Eq. (2.1). It is also patent that the SDP is less evident at high frequency, therefore low frequency is preferable in order to detect it.

In Fig. 5 (right) from $20 \mathrm{MHz}$ upwards, both formulas give very similar amplitudes for the electric field. However, at low frequencies, this is no longer the case. Both formulas predict a higher amplitude between 1 and $10 \mathrm{MHz}$ than in the rest of the spectrum, something that is in agreement with the low-frequency experiments carried out so far $[5,9,10]$.

\section{Outlook and conclusions}

We have shown that Eq. (2.1), with the appropriate charge and current density included, reproduces the electric field created by a particle track in the near-field regime. We have shown that its Fourier transform (Eq. (2.5)) is equivalent to an exact calculation of the field of a track [14]. Eq. (2.1) is useful if we want to calculate the electric field of a track at any frequency, in particular 

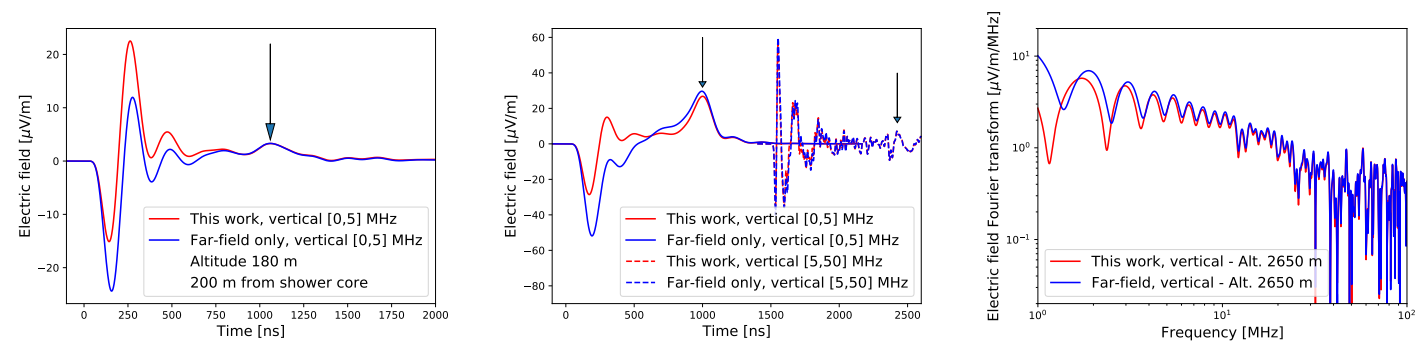

Figure 5: Left and centre: electric field (vertical polarisation) created by a $5 \mathrm{EeV}$ proton-induced $30^{\circ}$ shower for an antenna at $200 \mathrm{~m}$ East of the shower core. The SDP is visible after the principal pulse (indicated by the arrows) at $\sim 1000 \mathrm{~ns}$ after the first, negative pulse. Left: $180 \mathrm{~m}$ of altitude. Centre: $2650 \mathrm{~m}$ of altitude. A trace filtered between 5 and $50 \mathrm{MHz}$ is plotted, with time arbitrarily offset. Right: Fourier transform amplitude of the electric field (vertical).

below $5 \mathrm{MHz}$, corresponding to the near-field regime, as it is the case for the EXTASIS experiment [11].

We have implemented Eq. (2.1) in the SELFAS code and used it to calculate the electric field in the near-field regime created by an EAS. Differences with the far-field case are quite important for frequencies below $5 \mathrm{MHz}$ and more for the vertical component than the EW component. The SDP introduced in [5] is still present when calculating the near-field effects.

Nevertheless, since most of the difference between Eq. (2.1) and the far-field approach come from the existence of a longitudinally polarised component ( $\mathbf{r}$ in Eq. (2.1)) and since antenna responses are known usually for far-field only (without longitudinal component), further studies are needed in order to translate the electric field at the antenna into a measured voltage. A careful analysis of the antenna response, by means of simulations or measurements is needed for understanding our final data. A study of the influence of the ground on the electric field of an EAS is underway.

\section{Acknowledgements}

We thank Région Pays de la Loire for its financial support of the Groupe Astro of Subatech and in particular for their contribution to the EXTASIS experiment.

\section{References}

[1] F. G. Schröder. Progress in Particle and Nuclear Physics 93 (2017) 1-68.

[2] H. R. Allan. Progress in elementary particle and cosmic ray physics. North Holland (1971).

[3] G. A. Askaryan. J. Exp. Theor. Phy. 21 (1962) 658.

[4] A. Bellétoile et al. Astropart. Phys. 69 (2015) 50-60.

[5] B. Revenu, V. Marin. Proc. 33rd ICRC (2013).

[6] R. Dallier et al. See prooceedings for this conference.

[7] The Pierre Auger Collaboration. Phys. Rev. D 93 (2016) 122005. 
[8] W. D. Apel et al. Phys. Lett. B 763 (2016) 179.

[9] K. Nishi, K. Suga. Proc. 20th ICRC, Moscow, Russia 6 (1987) 125.

[10] C. Castagnoli, P. L. Ghia, F. Gomez and P. Trivero. Proc. 22nd ICRC, Dublin, Ireland 4 (1991) 363.

[11] A. Escudie et al. See proceedings for this conference.

[12] V. Marin, B. Revenu. Astroparticle Physics 35 (2012) 733-741.

[13] J. Alvarez-Muniz et al. Phys. Rev. D 81 (2010) 123009.

[14] D. García-Fernández et al. Phys. Rev D 87 (2013) 023003.

[15] F. Gaté et al. Computing the electric field from Extensive Air Showers using a realistic description of the atmosphere. Submitted to Astroparticle Physics.

[16] B. Revenu et al. See proceedings for this conference.

[17] P. Motloch et al. Proc 34th ICRC (2015) 448.

[18] O. Martineau-Huynh et al. Proc 34th ICRC (2015) 1143. 